\title{
Amnioprotective cesarean section in monochorionic- triamniotic triplet pregnancy, spontaneously conceived, complicated by intrahepatic cholestasis
}

\author{
Jarosław Kalinka, Maja Kufelnicka-Babout, tukasz Panasowiec \\ Department of Perinatology, First Chair of Obstetrics and Gynecology, Medical University of Lodz, Poland
}

Corresponding author: Łukasz Panasowiec, Department of Perinatology, First Chair of Obstetrics and Gynecology, Medical

University of Lodz, Poland, e-mail: lukaszpanasowiec@interia.pl

Triplet pregnancies from spontaneous conception are very rare. According to Hellin's rule: triplets $=\left(a^{2}\right)=1: 80^{2}$ $=1: 6400$ are $0.015 \%$ of all pregnancies (monochorionic-triamniotic triplet pregnancy occurs in $1: 100000$ ). They are at a high risk of pregnancy complications and have higher rates of perinatal morbidity and mortality, such as structural defects and neurodevelopmental anomalies.

A 30-year-old woman, gravida 2, para 2, was admitted to the $3^{\text {rd }}$ level perinatal center at 29 weeks in a monochorionic-triamniotic triplet pregnancy to observe fetal well-being. In past medical history removal of adrenal adenoma is worth mentioning. Previous pregnancy resulted in natural term birth of healthy male fetus weighing $3600 \mathrm{~g}$ in 2016.

Doppler ultrasound examination was performed and did not reveal any abnormalities in values either for middle cerebral or umbilical artery pulsatility index. This is shown in the graphs below (Figures $1 \mathrm{~A}, \mathrm{~B}$ ).

Cardiotocographic (CTG) records were performed every day. They did not reveal any abnormalities either.

At 30 weeks the patient reported itching of both hands that corresponded with the laboratory test results revealing cholestasis (AST 52,7 U/l, ALT 80,7 U/l). Bile acid level was $20 \mu \mathrm{mol} / \mathrm{l}$. Due to the onset of labor $(4 \mathrm{~cm}$ cervical dilatation) at 33 weeks of gestation and elevated liver enzyme levels, a caesarean delivery was indicated, resulting in live birth of triplets weighing $2050 \mathrm{~g}$ (AS 10), $1910 \mathrm{~g}$ (AS 9), $2050 \mathrm{~g}$ (AS 10). An APCS procedure (amnion protective caesarean section) was performed. The aim of this procedure is to remove the fetus in the entire amniotic sac, limiting the injury.

On the day after the surgery, the patient reported abdominal pain. Inflammatory parameters proved to be increased. An ultrasound examination revealed an inhomogeneous echogenic structure that might have corresponded to a hematoma $(131 \times 80 \times 117 \mathrm{~mm})$ in the lower part of the uterine muscle. Indeed, the hematoma was detected and removed during the next surgery. The inflammatory parameters decreased to normal values within the three following days (Figure 2).

The patient and newborns left the clinic on the $10^{\text {th }}$ day after the surgery in good health.

This is the third reported case of spontaneous monochorionic triamniotic triplets without fetal abnormalities or feto-fetal transfusion that was managed conservatively with an excellent health outcome for the mother and her three babies. This normal outcome may help in decision-making and parental counseling.

Monozygotic multiple gestations occur when a single fertilized ovum splits into genetically identical embryos. Exactly when this division occurs, it governs the configuration of chorion and amnion compartments. Monochorionic multiple pregnancies occur when the embryo undergoes cleavage 4-7 days after fertilization.

Cases of spontaneous trigeminal monochorionic triamniotic pregnancy are very rarely described [1-7]. It is well known that the prevalence of multiple pregnancies is directly 
A

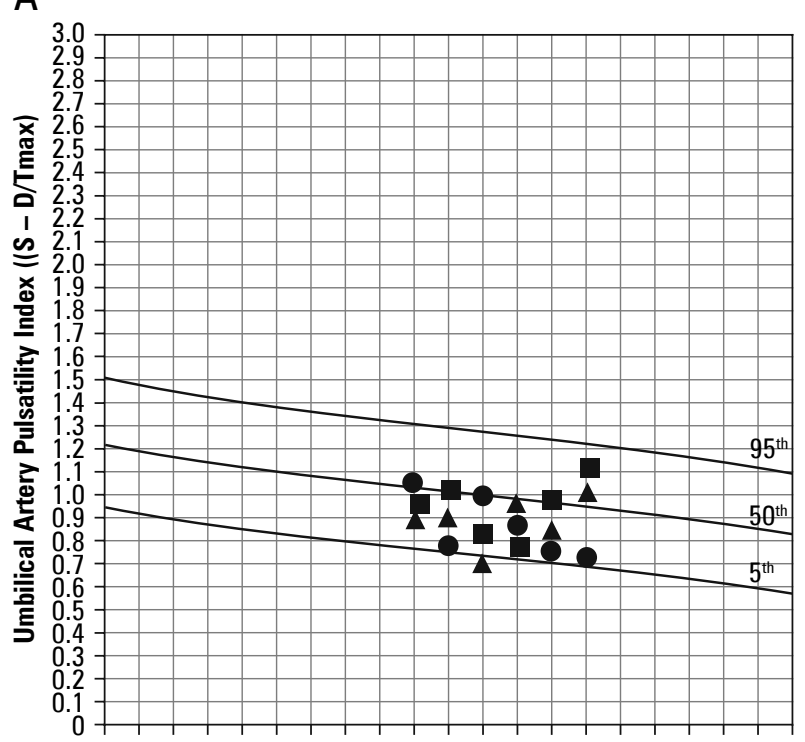

202122232425262728293031323334353637383940 Gestation [weeks]

- Triplet I $\Delta$ Triplet II $\quad$ Triplet III
B

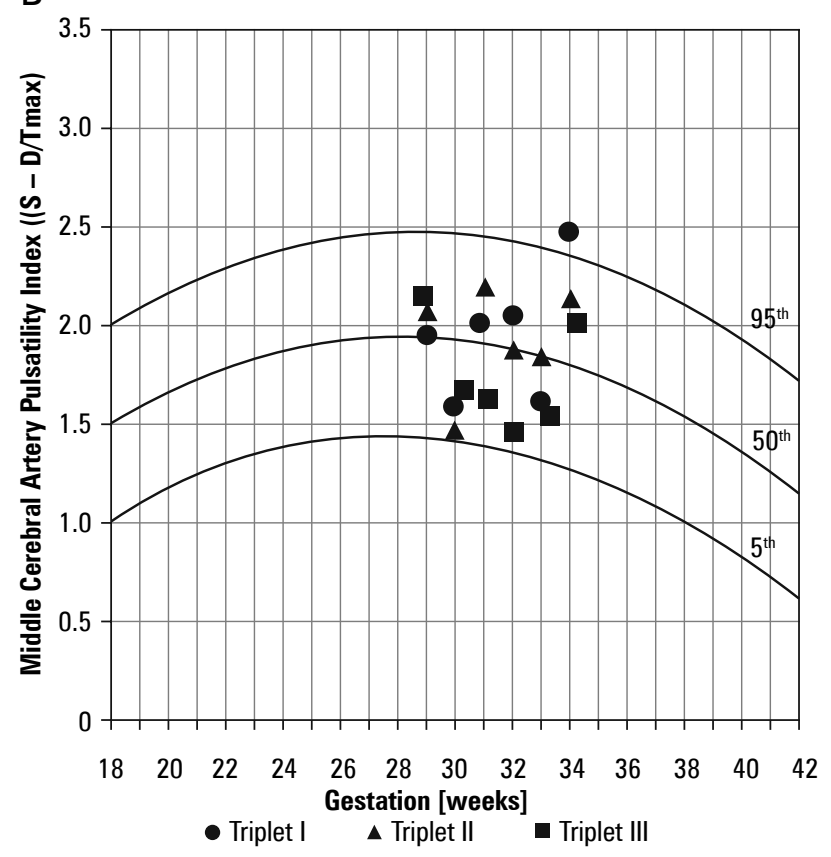

Figures 1. A, B - Comparison of fetal flow values in individual weeks

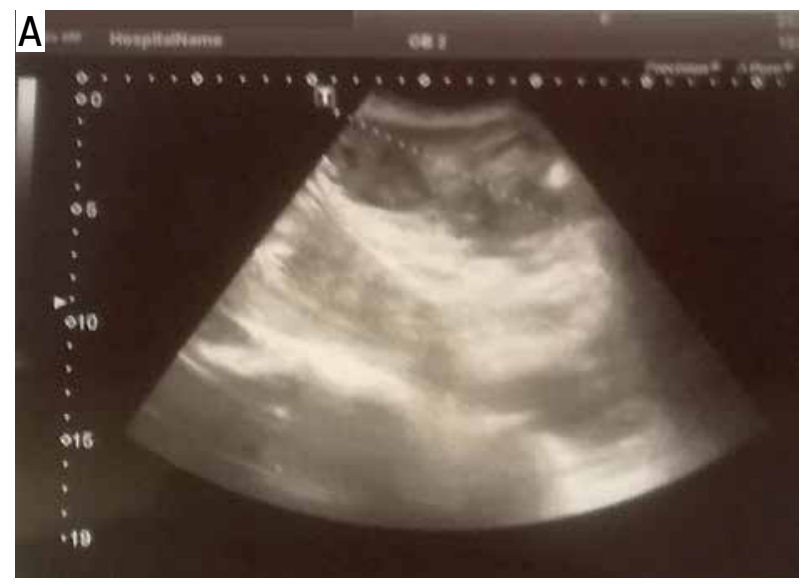

Figure 2. The hematoma $(131 \times 80 \times 117 \mathrm{~mm})$
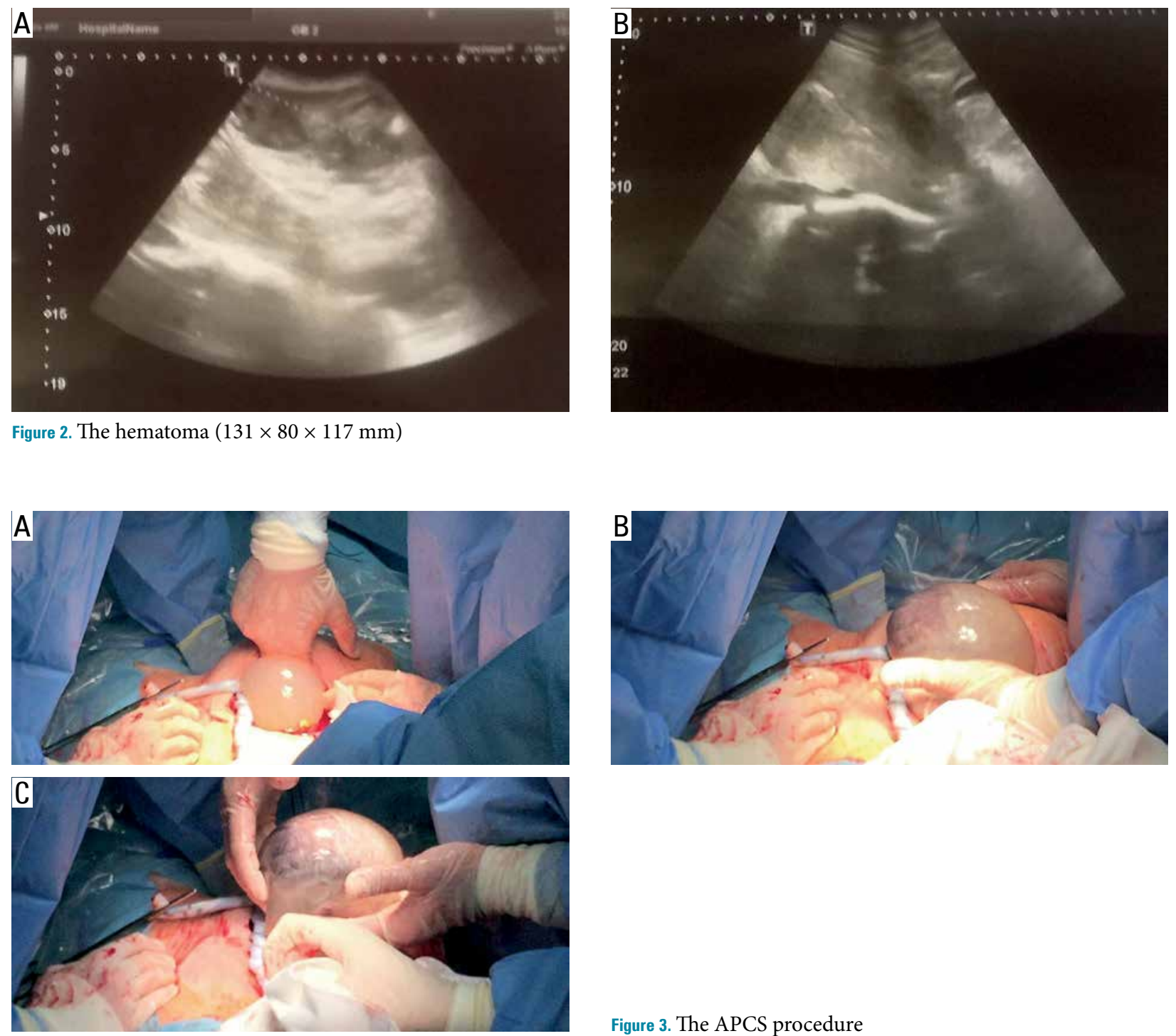

Figure 3. The APCS procedure 
related to the methods of in vitro fertilization [8]. Multiple pregnancies are also related to a higher incidence of maternal gestational co-morbidities (preeclampsia, gestational diabetes) then early and late abortions, twin-to-twin transfusion syndrome (TTTS) [9], intrauterine death of the fetuses and premature births, as well. Among multiple pregnancies the trigeminal ones could be complicated by fetal malformations and TTTS, which is possible to treat with fetoscopic laser coagulation, though with controversial perinatal outcomes [10-12].

There are only a few known cases of spontaneous monochorionic trigeminal pregnancy without fetal anomalies and TTTS described in the literature, pointing out that our case of spontaneously conceived monochorionic triamniotic pregnancy with a good pregnancy outcome is worth mentioning. To the best of our knowledge, there are only nine other reported cases of spontaneously conceived monochorionic triamniotic triplet pregnancies, three with congenital malformation in one of the triplets, whereas the other four developed feto-fetal transfusion with high perinatal morbidity and mortality, e.g. a twin reversed arterial perfusion (TRAP) sequence [13]. Among other described pathologies, there is also intrauterine growth restriction [14].

Interestingly, in our case study, maternal intrahepatic cholestasis (ICP) was brought on despite lack of any complications on the part of the fetus. Clinical evidence supports an etiologic role of estrogens in the initiation of ICP. ICP most commonly occurs in the last trimester, when estrogen levels reach their highest levels. ICP has been associated with twin and triplet pregnancies because of higher estrogen levels than in single gestations [15]. The prevalence of ICP was significantly higher in twin pregnancies as compared to single pregnancies (20.9\% vs. $4.7 \%)$ [16].

Last but not least, the birth weight of triplets needs further discussion. The triplets were in good health condition, in contrast to the other known cases with intrauterine fetal death [17]. Amnioprotective cesarean section (APCS) was performed and resulted in an atraumatic delivery. In the medical database there is no description of a similar APCS procedure in triplets (Figure 3).

Pregnancy of triplets as a type of multi-pregnancy entails a number of potential threats and risks. It should be emphasized that following such a pregnancy very close medical supervision is necessary. Health care for a pregnant woman and newborns ought to be provided by hospitals with the highest reference level. Doppler ultrasound examination as well as cardiotocographic records have to be performed on a daily basis. Multiple pregnancies require special medical attention so that any pathology may be promptly detected and dealt with. Medical doctors should be made aware of and ready for emergency termination of any high-risk pregnancy at all times. The APCS procedure should be widely advised during cesarean section.

\section{References}

1. Imaizumi Y. A coperative study of zygotic twinning and triplet rates in eight countries, 1972-1999. J Biosoc Sci 2003; 35: 287-302.
2. Ghulmiyyah LM, Perloe M, Tucker MJ, et al. Monochorionic-triamniotic triplet pregnancy after intracytoplasmic sperm injection, assisted hatching, and two-embryo transfer: first reported case following IVF. BMC Pregnancy Childbirth 2003; 3: 4.

3. Yanaihara A, Yorimitsu T, Motoyama H, et al. Monozygotic multiple gestation following in vitro fertili: analysis of seven cases from Japan. J Exp Clin Assist Reprod 2007; 4: 4.

4. Gul A, Aslan H, Cebeci A, et al. Monochorionic triamniotic triplet pregnancy with a co-triplet fetus discordant for congenital cystic adenomatoid malformation of the lung. Reprod Health 2005; 2: 2.

5. Iwamoto H, Yoshida A, Suzuki H, et al. Monochorionic triamniotic triplet pregnancies with assisted reproductive technology: two case reports. J Obstet Gynaecol Res 2010; 36: 872-5.

6. Gandham S, Ogueh O. Spontaneous monochorionic triplet pregnancy with no fetal anomaly or feto-fetal transfusion. BMJ Case Rep 2012; 2012: pii: bcr2012007114. doi: 10.1136/bcr-2012007114.

7. Habek D, Marton I, Luetić AT, et al. Spontaneous monochorionic triamniotic triplet pregnancy with good perinatal outcome. Ann Womens Health 2017; 1: 1004.

8. Gurunath S, Makam A, Vinekar S, Biliangady RH. Monochorionic triamniotic triplets following conventional in vitro fertilization and blastocyst transfer. J Hum Reprod Sci 2015; 8: 54-7.

9. Pan P, Luo G, Tang L, et al. Monochorionic-triamniotic triplet pregnancy complicated by twin reversed arterial perfusion sequence: case report and literature review. Am J Perinatol Rep 2017; 7: e106-10.

10. Entezami M, Runkel S, Becker R, et al. Feto-feto-fetal triplet transfusion syndrome (FFFTTS). J Matern Fetal Med 1997; 6: 334-7.

11. Baschat AA, Muench MV, Mighty HE, Harman CR. Successful intrauterine management of severe feto-fetal transfusion in a monochorionic triplet pregnancy using bipolar umbilical cord coagulation. Fetal Diagn Ther 2003; 18: 397-400.

12. Saravelos SH, Zhang T, Chung JP, et al. Monochorionic quadramniotic and triamniotic pregnancies following single embryo transfers: two case reports and a review of the literature. J Assist Reprod Genet 2016; 33: 27-32.

13. Argoti PS, Bebbington MW, Johnson A, Moise KJ Jr. Indirect pump: unique presentation of a monochorionic-trianiotic triplet gestation complicated by TRAP sequence and succesfully managed with radiofrequency ablation of the acardiac fetus. Ultrasound $\mathrm{Ob}-$ stet Gynecol 2013; 42: 115-7.

14. López-Pérez R, Lorente M, Martínez-Uriarte J, et al. Twin-reserved arterial perfusion sequence in a triple monochorionic pregnancy with two direct pump fetuses results in significant cyclic Doppler waveform. Fetal Diagn Ther 2015; 37: 157-60.

15. Pusl T, Beuers U. Intrahepatic cholestasis of pregnancy. Orphanet J Rare Dis 2007; 2: 26.

16. Gonzalez MC, Reyes H, Arrese M, et al. Intrahepatic cholestasis of pregnancy in twin pregnancies. J Hepatol 1989; 9: 84-90.

17. Imaizumi Y. Perinatal mortality in triplet births in Japan: time trends and factors influencing mortality. Twin Res 2003; 6: 1-6. 